\title{
THE DEPENDENCE OF THE GENERALIZED RADON TRANSFORM ON DEFINING MEASURES
}

BY

ERIC TODD QUINTO

\begin{abstract}
Guillemin proved that the generalized Radon transform $R$ and its dual $R^{t}$ are Fourier integral operators and that $R^{t} R$ is an elliptic pseudodifferential operator. In this paper we investigate the dependence of the Radon transform on the defining measures. In the general case we calculate the symbol of $R^{\prime} R$ as a pseudodifferential operator in terms of the measures and give a necessary condition on the defining measures for $R^{t} R$ to be invertible by a differential operator. Then we examine the Radon transform on points and hyperplanes in $\mathbf{R}^{n}$ with general measures and we calculate the symbol of $R^{t} R$ in terms of the defining measures. Finally, if $R^{t} R$ is a translation invariant operator on $R^{n}$ then we prove that $R^{t} R$ is invertible and that our condition is equivalent to $\left(R^{t} R\right)^{-1}$ being a differential operator.
\end{abstract}

Introduction. In the first part of this century Radon [18] created the classical Radon transform on points and lines in $\mathbf{R}^{2}$. From this start others invented new Radon transforms of a similar nature. Helgason has studied many Radon transforms (e.g. [12]; [10] summarizes his early work) and has provided a framework in which to define Radon transforms on homogeneous spaces and more generally. Gelfand [3] first defined the topological concept of double fibration that yields Radon transforms quite generally. Given manifolds $X$ and $Y$ related by a double fibration, a class of submanifolds of $X$ indexed by elements of $Y,\left\{H_{y} \mid y \in Y\right\}$, is defined. The Radon transform $R: C_{0}^{\infty}(X) \rightarrow C_{0}^{\infty}(Y)$ takes $f \in C_{0}^{\infty}(X)$ to the integrals of $f$ over the $H_{y}$ with respect to measures derived from given measures on $X, Y$, and $Z$. A dual transform $R^{t}: C^{\infty}(Y) \rightarrow C^{\infty}(X)$ is also defined. In special cases $R^{t} R$ has been shown to be invertible, often by a differential operator. Invertible Radon transforms have nice applications (e.g. [2], [13], [16]) and if $\left(R^{t} R\right)^{-1}$ is a differential operator, less information is needed to invert $R$ than if it is not. In the general case of Gelfand's under an added hypothesis, the Bolker assumption, Guillemin [7], [8] has shown that $R$ and $R^{t}$ are Fourier integral operators and that $R^{t} R$ is an elliptic pseudodifferential operator. Therefore $R^{t} R$ is locally invertible.

$\$ 1$ of this article lays the groundwork for the later parts. We explain the double fibration and define the generalized Radon transform and its dual. Finally we show how the classical Radon transform on points and hyperplanes in $\mathbf{R}^{n}$ fits into this framework.

Received by the editors January 17, 1979.

AMS (MOS) subject classifications (1970). Primary 44A05; Secondary 58G15.

Key words and phrases. Generalized Radon transforms, Radon transforms on $\mathbf{R}^{n}$, smooth positive measure, pseudodifferential operator, symbol of pseudodifferential operator. 
The role that the defining measures play in the general Radon transform has never been studied; one goal of this paper is to better understand this role. In \$2 we calculate, under the Bolker assumption (9), the symbol of $R^{t} R$ as a pseudodifferential operator and show its dependence on the defining measures (Theorem 2.1). This gives a necessary condition on the defining measures for $R^{t} R$ to be invertible by a differential operator (Theorem 2.2). If $R^{t} R$ is invertible by a differential operator and if $f \in C_{0}^{\infty}(X)$, one only needs to know integrals of $f$ over submanifolds $H_{y}$ that are "infinitesimally close" to $x$ to recover the value of $f(x)$ from the values of $R f$. So if $\left(R^{t} R\right)^{-1}$ is a differential operator, "less information" is needed to invert the Radon transform than if it is not.

Another goal of this paper is to understand the general results of Theorems 2.1 and 2.2 for the Radon transform on points and hyperplanes in $\mathbf{R}^{n}$ with general measures. In $\$ 3$ we calculate the symbol of $R^{t} R$ explicitly in terms of the defining measures, illustrating the necessary condition of Theorem 2.2 for this case (Theorem 3.1). Finally in $\$ 4$ we study the class of translation invariant Radon transforms on $\mathbf{R}^{n}$. These transforms are natural generalizations of the classical Radon transform on $\mathbf{R}^{n}$ which is both translation and rotation invariant. We show that they are invertible and for such a transform $\left(R^{t} R\right)^{-1}$ is a differential operator if and only if the condition of Theorem 2.2 is satisfied (Theorem 4.2).

The author would like to thank his thesis advisor, Victor Guillemin, for his inspiration and guidance as well as his astute insights on the Radon transform. The author also thanks Richard Melrose and the reviewer for their many helpful suggestions about the article.

1. Double fibrations. We first define the notion of double fibration due to Gelfand [3] and from this define $R$ and $R^{t}$. Then we examine the classical Radon transform on points and hyperplanes in $\mathbf{R}^{n}$ and show how it fits into this framework.

Let $X$ and $Y$ be connected paracompact $C^{\infty}$ manifolds, each of dimension $n$. Let $Z$ be a closed submanifold of $X \times Y$ of codimension $k, k>0$. Call $\pi$ and $\rho$ the projections to $X$ and $Y$, respectively. We assume that both $\pi$ and $\rho$ are fiber mappings with connected fibers, and that $\pi$ is proper.

For each $y \in Y$, the set $H_{y}=\pi \rho^{-1}\{y\}=\{x \in X \mid(x, y) \in Z\}$ is a closed codimension $k$ submanifold of $X$ diffeomorphic to the fiber $\rho^{-1}\{y\}$. Similarly, for each $x \in X, G_{x}=\rho \pi^{-1}\{x\}=\{y \in Y \mid(x, y) \in Z\}$ is a closed codimension $k$ submanifold of $Y$ diffeomorphic to $\pi^{-1}\{x\}$.

Throughout the article we assume

(i) $G_{x_{1}}=G_{x_{2}}$ if and only if $x_{1}=x_{2}$,

(ii) $\quad H_{y_{1}}=H_{y_{2}}$ if and only if $y_{1}=y_{2}$.

This assures that each point $x \in X$ corresponds to a unique manifold $G_{x}$ and each $y \in Y$ corresponds to a unique $H_{y}$.

To summarize, we have:

Definition 1.1. Let $X$ and $Y$ be $C^{\infty}$ manifolds each of dimension $n$, and let $Z$ be a closed codimension $k$ submanifold of $X \times Y(k>0)$. 
Assume

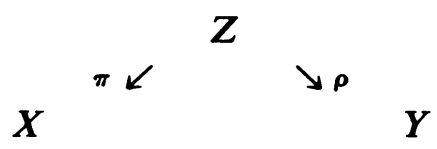

is a pair of fiberings with connected fibers. If $\pi$ is proper and (1) is satisfied, then (2) is called a double fibration.

This idea was first developed by Gelfand [3] with no restrictions on $\operatorname{dim} X$ and $\operatorname{dim} Y$.

A double fibration will define two integral transforms. Before defining them we will discuss measures. Let $\pi: U \rightarrow V$ be a fibering of manifolds and let $\mu$ be a smooth measure (i.e., smooth with respect to Lebesgue measure on each coordinate patch) of compact support on $U$. The push forward of $\mu, \pi_{*} \mu$, is defined to be the unique measure on $V$ satisfying

$$
\int_{V} f \pi_{*} \mu=\int_{U}\left(\pi^{*} f\right) \mu
$$

for all $f \in C_{0}^{\infty}(V)$. The smooth measure on $V, \pi_{*} \mu$, is the result of integrating $\mu$ along the fibers of $U \rightarrow V$. If $\pi$ is proper, $\pi_{*} \mu$ can be defined for any $C^{\infty}$ measure $\mu$.

Our next definition is due to Guillemin [7].

DEFINITION 1.2. Let (2) be a double fibration with smooth, positive nowhere zero measures $\mu, m$, and $n$ given on $Z, X$, and $Y$, respectively. The Radon transform $R$ : $C_{0}^{\infty}(X) \rightarrow C_{0}^{\infty}(Y)$ is defined by the relation

$$
R f n=\rho_{*}\left(\pi^{*} f \mu\right) \text { for each } f \in C_{0}^{\infty}(X) .
$$

The dual transform $R^{t}: C^{\infty}(Y) \rightarrow C^{\infty}(X)$ is defined by

$$
R^{t} g m=\pi_{*}\left(\rho^{*} g \mu\right) \text { for } g \in C^{\infty}(Y) \text {. }
$$

As $\pi$ is proper, $\left(\pi^{*} f\right) \mu$ is a smooth measure of compact support on $Z$ and so $R f \in C_{0}^{\infty}(Y)$ is well defined by (3). Similar reasoning shows $R^{t}$ is also well defined.

Throughout this article $R$ will denote a Radon transform in the sense of Definition 1.2; $R^{t}$ will be its dual. The measures $\mu, m$, and $n$ will often be called defining measures.

We now describe the Radon transform more concretely. The defining measures determine a measure on $H_{y}$; for each $y \in Y$ one can identify the fiber of $Z$ above $y, \rho^{-1}\{y\}$, with $H_{y}$ and so the measure $\mu$ on $Z$ can be written as a product of the measure $n$ on $Y$ and a measure $\mu_{y}$ on $H_{y}$. If $f \in C_{0}^{\infty}(X)$, then $\pi^{*} f \mu=f(x) \mu_{y} n$ and

$$
R f(y)=\int_{H_{y}} f(x) d \mu_{y}(x)
$$

Similarly, each $G_{x}$ acquires a measure $\mu_{x}$, and, for $g \in C^{\infty}(Y)$,

$$
R^{t} g(x)=\int_{G_{x}} g(y) d \mu_{x}(y)
$$


This shows that $R$ and $R^{t}$ are integrations over the submanifolds $H_{y}$ and $G_{x}$ that come from the double fibration; the measures $\mu_{y}$ and $\mu_{x}$ are defined by pushing $\mu$ forward to $Y$ and $X$, respectively.

Our first proposition will be useful for calculating the symbol of $R^{t} R$.

Proposition 1.1. Viewed as distributions on $X \times Y$ with measure $m n$, the Schwartz kernels of $R$ and $R^{t}$ are both equal to the functional taking $h \in C_{0}^{\infty}(X \times$ Y) to $\int_{z} h(x, y) d \mu$.

Proof. Let $f \in C_{0}^{\infty}(X)$ and $g \in C_{0}^{\infty}(Y)$. If $K$ is the Schwartz kernel of $R$,

$$
K\left(\pi^{*} f \rho^{*} g\right)=\int_{Y} R f(y) g(y) d n(y)=\int_{Z} \pi^{*} f \rho^{*} g d \mu
$$

by the definition of $R$. As sums of products $\pi^{*} f \rho^{*} g$ are dense in $C_{0}^{\infty}(X \times Y)$, $K=\int_{Z} \cdot d \mu$. The proof for $R^{t}$ is similar.

A variation on this proof shows that $R^{t}$ is formally dual to $R$ with measures $m$ on $X$ and $n$ on $Y$.

We now look at a classical example and show how it fits into this framework. Example 1 is the best known Radon transform and was first studied by Radon [18] and John [15] in the early part of this century.

EXAMPLE 1. The classical Radon transform on points and hyperplanes in $\mathbf{R}^{n}$.

Let $X$ be $\mathbf{R}^{n}$ and let - be the standard inner product on $\mathrm{R}^{n}$. Let $Y$ be the space of $(n-1)$-dimensional hyperplanes in $\mathbf{R}^{n}$. If $\omega \in \mathbf{S}^{n-1}$ and $p \in \mathbf{R}$, then the hyperplane $H(\omega, p)=\left\{x \in \mathbf{R}^{n} \mid x \cdot \omega=p\right\}$ has normal vector $\omega$ and directed distance $p$ from the origin in $\mathbf{R}^{n}$. Because $H(\omega, p)=H(-\omega,-p), Y$ can be identified with $\left(\mathbf{S}^{n-1} \times \mathbf{R}\right) / \mathbf{Z}_{2}$; let $[\omega, p]$ denote the projection of $(\omega, p) \in \mathbf{S}^{n-1} \times \mathbf{R}$. Define $(x,[\omega, p])$ to be in $Z$ if and only if $x \in H(\omega, p)$; then

$$
Z=\left\{(x,[\omega, x \cdot \omega]) \mid x \in \mathbf{R}^{n}, \omega \in \mathbf{S}^{n-1}\right\}
$$

and we can identify $Z$ with $\mathbf{R}^{n} \times \mathbf{R P}^{n-1}$. With these choices for $X, Y$, and $Z$, (2) is a double fibration. The submanifold $G_{x}=\rho \pi^{-1}\{x\}$ is $\left\{[\omega, x \cdot \omega] \mid \omega \in \mathbf{S}^{n-1}\right\}$ and represents the set of hyperplanes that pass through $x$. We identify $G_{x}$ with $\mathbf{R P}^{n-1}$.

Let $d x$ and $d p$ be Lebesgue measures on $\mathbf{R}^{n}$ and $\mathbf{R}$, respectively, and let $d \omega$ be the measure on $\mathbf{R P}^{n-1}$ induced from its standard Riemannian metric. Let $d \omega d p$ be the measure on $Y$ induced from the standard Riemannian metric on $S^{n-1} \times \mathbf{R}$; locally this is the product of $d \omega$ and $d p$ defined above. Finally we choose the measure $d x d \omega$ on $Z \cong \mathbf{R}^{n} \times \mathbf{R P}^{n-1}$. These measures define a Radon transform and its dual. This is essentially the classical Radon transform on $\mathbf{R}^{n}$.

For $[\omega, p] \in Y$ the resulting measure $\mu_{\{\omega, p]}$ on the hyperplane $H(\omega, p)$ is Lebesgue measure and, for $f \in C_{0}^{\infty}\left(\mathbf{R}^{n}\right), R f[\omega, p]$ is the integral of $f$ over $H(\omega, p)$ with respect to this measure. The measure $\mu_{x}$ on $G_{x} \cong \mathbf{R P}^{n-1}$ is $d \omega$ and $R^{t}$ integrates with respect to this measure over $G_{x}$.

The following theorem is a consequence of Theorem 4.2 and has been proven in different forms by Radon [18], John [15], Gelfand [4], Helgason [11], Ludwig [16] and Semyanistyi [20]. 
THEOREM 1.2. For the classical Radon transform on $\mathbf{R}^{n}, R^{t} R$ is an elliptic pseudodifferential operator and is invertible by an operator $L$. If $n$ is odd,

$$
L=(2 \pi i)^{1-n}(\Delta)^{(n-1) / 2}
$$

where $\Delta$ is the Laplacian on $\mathbf{R}^{n}$. For even $n, L$ is not a differential operator.

2. The symbol of $R^{t} R$ in the general case. Under a nondegeneracy condition (the Bolker assumption (9)), the symbol of $R^{t} R$ is calculated in terms of the defining measures using the calculus of Fourier integral operators (Theorem 2.1). From this a necessary condition for $R^{t} R$ to be invertible by a differential operator is obtained in terms of the defining measures (Theorem 2.2).

We now discuss the Bolker assumption. Let

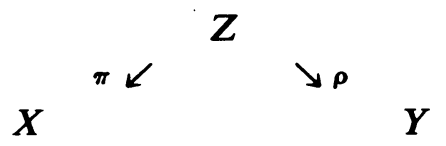

be a double fibration and let $\Gamma$ denote $N^{*} Z-0$, the conormal bundle of $Z$ with its 0 section removed. Then $\Gamma$ is a closed, conic, Lagrangian submanifold of $T^{*}(X \times$ $Y)-0$. Consider the projections

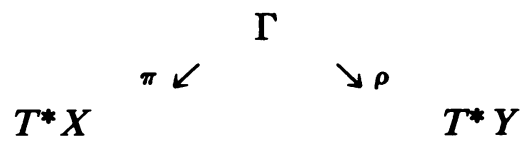

We assume

$$
\rho: \Gamma \rightarrow T^{*} Y \text { is an injective immersion. }
$$

This is called the Bolker assumption because Ethan Bolker stated a similar assumption for finite Radon transforms [7]. Since $\Gamma$ is Lagrangian, $\pi$ must also be a local diffeomorphism linear in the fibers of $\Gamma \rightarrow Z$ and so $\Gamma \subset\left(T^{*} X-0\right) \times\left(T^{*} Y\right.$ $-0)$. Because $\pi: Z \rightarrow X$ is proper, one can show that $\pi: \Gamma \rightarrow T^{*} X-0$ is either a 2-to-1 cover or a diffeomorphism if $\operatorname{dim} X>2$ (finite-to-one if $\operatorname{dim} X=2$ ) [17]. This requirement places severe restrictions on the topology of $G_{x}$ and the codimension $k$ of $Z$ in $X \times Y(k=1,2,4$, or 8) [17].

REMARK. In [7] Guillemin proves that $R^{t} R$ is an elliptic pseudodifferential operator under the assumption that $\operatorname{dim} X<\operatorname{dim} Y$. Throughout this article we assume that $\operatorname{dim} X=\operatorname{dim} Y$; this makes the computation of the symbol of $R^{t} R$ easier.

The goal of our present discussion is to calculate the symbol of $R^{t} R$ as a pseudodifferential operator. We will need to sketch a proof of Guillemin's result ([7], [8]) that $R^{t} R$ is an elliptic pseudodifferential operator of order $k$ - $\operatorname{dim} X$ ( $k=\operatorname{codim} Z$ in $X \times Y$ ) but will only detail the parts relevant to calculating the symbol of $R^{t} R$.

First we present some preliminaries on Lagrangian distributions and Fourier integral operators as in [8] or [14]. Let $S$ be a manifold; let $|\Lambda|^{1 / 2} S$ be the space of smooth half densities on $S$, and let $|\Lambda|_{0}^{1 / 2} S$ be those of compact support. Give these spaces the usual topologies [14]. Let $\left(|\Lambda|^{1 / 2} S\right)^{\prime}$ be the dual space of $|\Lambda|^{1 / 2} S$ and let $\left(|\Lambda|_{0}^{1 / 2} S\right)^{\prime}$ be the dual space of $|\Lambda|_{0}^{1 / 2} S$. Let $\Gamma \subset T^{*} S-0$ be a closed, conic, 
Lagrangian submanifold and let $I^{r}(\Gamma) \subset\left(|\Lambda|_{0}^{1 / 2} S\right)^{\prime}$ be the set of Lagrangian distributions of order $r$ and type 1, 0 associated to $\Gamma$ ([14, Definition 3.2.2]). If $X$ and $Y$ are manifolds of dimension $n$, let $C$ be a local canonical graph in $\left(T^{*} X-0\right) \times$ $\left(T^{*} Y-0\right)$ and define

$$
C^{\prime}=\left\{(x, \xi, y, \eta) \in T^{*} X \times T^{*} Y \mid(x, \xi, y,-\eta) \in C\right\} .
$$

An operator $\bar{V}:|\Lambda|_{0}^{1 / 2} X \rightarrow|\Lambda|^{1 / 2} Y$ is in $\overline{F r}^{r}(C)$ if the Schwartz kernel, $\nu$, of $\bar{V}$ is in $I^{r}\left(C^{\prime}\right)$. We will follow the conventions in [14] for defining $\sigma(\nu)$ and $\sigma(\bar{V})$, the principal symbols of $\nu$ and $\bar{V}$, respectively.

First a distribution related to the Radon transform is investigated. Let $X, Y$ and $Z$ be manifolds that define a double fibration satisfying (9) and let $n=\operatorname{dim} X$ and $k=\operatorname{codim} Z$ in $X \times Y$. Specify defining measures $\mu, m$, and $n$ on $Z, X$, and $Y$, respectively. These measures define a Radon transform $R$ and its dual $R^{t}$. Let $\Gamma$ denote $N^{*} Z-0$, the conormal bundle of $Z$ in $X \times Y$ with its zero section removed.

Let $I_{Z} \in\left(|\Lambda|_{0}^{1 / 2} X \times Y\right)^{\prime}$ be defined by

$$
I_{Z}(f \sqrt{m n})=\int_{Z} f d \mu
$$

for $f \in C_{0}^{\infty}(X \times Y)$. Here $\sqrt{m n}$ is the half density on $X \times Y$ defined from the product measure $m n$.

We now calculate the symbol of $I_{z} \in I^{(k-n) / 2}(\Gamma)$. Because $\Gamma$ is the conormal bundle of a closed submanifold we can choose phase functions linear in the phase variables ("linear phase functions") to define $I_{z}$ locally; then using these phase functions $\sigma\left(I_{Z}\right)$ is invariantly defined as a half density on $\Gamma$ modulo lower order half densities [14, Theorem 2.4.2] and the Maslov bundle of $\Gamma$ is trivialized canonically [14, Theorem 3.3.4].

Let $S$ be an open set in $X \times Y$ that intersects $Z$ on which local coordinates

$$
\left(z_{1}, \ldots, z_{2 n-k}, w_{1}, \ldots, w_{k}\right) \in \mathbf{R}^{2 n}
$$

are given such that $Z \cap S$ is the slice $w_{1}=0=\cdots=w_{k}$. Let $s \in S$ then $s=(z, w)$ where $z=\left(z_{1}, \ldots, z_{2 n-k}\right), w=\left(w_{1}, \ldots, w_{k}\right)$. Let $\zeta_{i}=\mathrm{dz}_{i}, i=$ $1, \ldots, 2 n-k$, and let $\eta_{j}=\mathbf{d w}_{j}, j=1, \ldots, k$, then $(z, w, \zeta, \eta)$ defines coordinates on $T^{*} S$ such that

$$
\Gamma \cap T^{*} S=\left\{\left(z, 0, \sum_{j=1}^{k} \theta_{j} \eta_{j}\right) \mid \theta \in \mathbf{R}^{k}-0, z \in \mathbf{R}^{2 n-k}\right\} .
$$

Let $\sqrt{d \eta}$ be the half density on the fiber of $\Gamma \cap T^{*} S$ satisfying $\sqrt{d \eta}\left(\partial / \partial \eta_{1}, \ldots, \partial / \partial \eta_{k}\right)=1$. Define $\sqrt{d z}$ and $\sqrt{d w}$ similarly and then $d z$ is a measure on $Z$ and $d z d w$ is Lebesgue measure on $S$. Finally define the functions $\mu(z)$ and $m(z, w)$ by requiring $\mu=\mu(z) d z$ and the product measure $m n=$ $m(z, w) d z d w$.

It is a straightforward exercise to show for $f \in C_{0}^{\infty}(S)$ that $I_{Z}(f \sqrt{m n})=(2 \pi)^{-(n+k) / 2}$

$$
\times \int_{R^{2 n-k} \times R^{2 k}} e^{i w \cdot \theta}(2 \pi)^{(n-k) / 2} \frac{\mu(z)(f(z, w) \sqrt{m(z, w)}) d w d \theta d z}{\sqrt{m(z, 0)}}
$$


and so $I_{Z}$ is a Lagrangian distribution with linear phase function

$$
\varphi(s, \theta)=w \cdot \theta
$$

and amplitude $(2 \pi)^{(n-k) / 2} \mu(z) / \sqrt{m(z, 0)}$ locally. One can now see that $I_{Z} \in$ $I^{(k-n) / 2}(\Gamma)$.

Let $d \varphi_{s}$ (resp., $d \varphi_{\theta}$ ) be the differential of $\varphi$ with respect to the $s$ variables (resp., the $\theta$ variables) and let

$$
\begin{aligned}
C_{\varphi} & =\left\{(z, w, \theta) \in \mathbf{R}^{2 n-k} \times \mathbf{R}^{k} \times \mathbf{R}^{k} \mid \theta \neq 0, d \varphi_{\theta}(z, w, \theta)=0\right\} \\
& =\left\{(z, 0, \theta) \mid z \in \mathbf{R}^{2 n-k}, \theta \in \mathbf{R}^{k}-0\right\} .
\end{aligned}
$$

Then, above $S, \Gamma$ is defined by the map

$$
\Phi: C_{\varphi} \rightarrow T^{*} S, \quad \Phi(z, 0, \theta)=\left(z, 0, d \varphi_{s}(z, 0, \theta)\right) .
$$

Let $d_{\varphi}$ be the pullback of the Dirac measure at $0 \in \mathbf{R}^{k}$ under the map $C_{\varphi} \rightarrow \mathbf{R}^{k}$, $(z, 0, \theta) \rightarrow d \varphi_{\theta}(z, 0, \theta)$, then in $\left(\left[14\right.\right.$, p. 118]) the symbol $\sigma\left(I_{Z}\right)$ for $I_{Z}$ is locally

$$
\Phi_{*}\left((2 \pi)^{(n-k) / 2} \mu(z) \sqrt{d_{\varphi}} / \sqrt{m(z, 0)}\right) .
$$

It is a standard exercise $([14$, p. 118]) to show that this pushforward is

$$
\begin{aligned}
\sigma\left(I_{Z}\right) & =\frac{(2 \pi)^{(n-k) / 2} \mu(z) \sqrt{d z} \sqrt{d \eta}}{\sqrt{m(z, 0)}} \\
& =\frac{(2 \pi)^{(n-k) / 2} \mu \sqrt{d w} \sqrt{d \eta}}{\sqrt{m n}} .
\end{aligned}
$$

Because each phase function (13) that locally defines $I_{Z}$ is linear and the local expressions (14) are all homogeneous, they fit together exactly to a half density on $\Gamma$.

We can now calculate the symbol of $R^{t} R$. Let $C=\Gamma^{\prime}$ and let $C^{t}$ be $C$ with the $T^{*} X$ and $T^{*} Y$ coordinates reversed. By the Bolker assumption (9), $C$ is a local canonical graph and so the distribution $I_{Z}$ is the Schwartz kernel of an operator $\bar{R} \in \overline{F I}^{(k-n) / 2}(C)$ as well as $\bar{R}^{t} \in \overline{F I}^{(k-n) / 2}\left(C^{t}\right)$. The symbol $\sigma(\bar{R})$ for $\bar{R}$ is the function $\sigma\left(I_{Z}\right) / \pi^{*}\left|\sigma_{X}\right|^{n / 2}$ pulled back to $C ; \pi$ is the projection from $\Gamma$ to $T^{*} X-0$, $\sigma_{X}$ is the canonical symplectic form on $T^{*} X$, and $\left|\sigma_{X}\right|^{n / 2}$ is the half density $\left|\sigma_{X}^{n} / n !\right|^{1 / 2}$ ([14, p. 169]; because $\sigma\left(I_{Z}\right)$ can be defined invariantly without the Maslov bundle, so can $\sigma(\bar{R}))$. The symbol $\sigma\left(\bar{R}^{t}\right)$ is defined in a similar manner.

Let $\Delta_{X}$ (resp., $\Delta_{Y}$ ) be the diagonal in $\left(T^{*} X-0\right)^{2}$ (resp., $\left.\left(T^{*} Y-0\right)^{2}\right)$. To compute a symbol for $\bar{R}^{t} \bar{R}$ multiply $\sigma(\bar{R})$ and $\sigma\left(\bar{R}^{t}\right)$ together on

$$
\Lambda=\left(C \times C^{t}\right) \cap\left(T^{*} X \times \Delta_{Y} \times T^{*} X\right)
$$

and project to $\left(T^{*} X\right)^{2}$ [8, Proposition 6.2, p. 359]. By (9) the projection of $\Lambda$ is $\Delta_{X}$ and $\Lambda$ can be identifed with $\Gamma$; we identify $\Delta_{X}$ with $T^{*} X-0$. Using the local expression (14) gives a symbol for $\bar{R}^{t} \bar{R}$ on an open subset $V$ of $T^{*} X-0$.

$$
\frac{(2 \pi)^{n-k} \mu^{2} d w d \eta}{m n \pi^{*}\left|\sigma_{X}\right|^{n / 2} \rho^{*}\left|\sigma_{Y}\right|^{n / 2}}\left(\pi^{-1}(x, \xi)\right) \text {. }
$$


Here $\pi$ is the projection from $\Gamma$ to $T^{*} X-0$ and the densities $d w$ and $d \eta$ are those of (11). If $\pi: \Gamma \rightarrow T^{*} X-0$ is 2-to-1 then (15) is the sum of $\sigma(\bar{R}) \otimes \sigma\left(\bar{R}^{t}\right)$ at the two pre-images.

Using Proposition 1.1 and the definition (10) of $I_{Z}$ one can see that $R^{t} R f=$ $\bar{R}^{t} \bar{R}(f \sqrt{m}) / \sqrt{m}$ for $f \in C_{0}^{\infty}(X)$ and so the classical symbol of $R^{t} R$ on $V$ is a factor of $\exp (\pi i \sigma / 4)$ times (15) $([14$, Theorem 3.2.1]) where $\sigma([14,(3.2 .10)])$ is an integer related to any linear phase function for pseudodifferential operators on $V^{2}$ as well as the "product" phase function that defines (15) from (12), (13) and (14) ([14, p. 176ff]). One can calculate from $([14,(3.2 .10)])$ that $\sigma$ is zero for these phase functions and so (15) is exactly the classical symbol of $R^{t} R$.

We have proven:

THEOREM 2.1. Assume the double fibration (7) satisfies the Bolker assumption (9). Then the symbol of $R^{t} R$ as a pseudodifferential operator is given by (15) where the densities $d w$ and $d \eta$ are defined below (11).

We now see one reason to know whether $R^{t} R$ is invertible by a differential operator. Let $f \in C_{0}^{\infty}(X)$. Recall from (5) that $R f(y)$ integrates $f$ over the manifold $H_{y}$. The set $G_{x}$ is precisely the set of $y$ such that $H_{y}$ passes through $x$. From the integral (6) we see that $R^{t} R f(x)$ is a weighted average of the integrals of $f$ over all $H_{y}$ that pass through $x$. If $R^{t} R$ is invertible by a differential operator-a local operator-then one only needs to know the integrals of $f$ over manifolds $H_{y}$ that are "infinitesimally close" to $x$ in order to recover $f(x)$ from values of $R f$. This greatly reduces the information needed to invert the Radon transform.

In general, an elliptic pseudodifferential operator $A$ is locally invertible by a pseudodifferential operator $B$ with $\sigma(B)=1 / \sigma(A)$; so if $B$ is a differential operator $1 / \sigma(A)$ must be a polynomial. This proves our next theorem.

THEOREM 2.2. Assume the double fibration (7) satisfies the Bolker assumption. If $1 / \sigma\left(R^{t} R\right)$ is not a polynomial on $T^{*} X$ then $R^{t} R$ is not invertible by a differential operator.

Because (15) gives $\sigma\left(R^{t} R\right)$ in terms of the defining measures, Theorem 2.2 gives a necessary condition on the measures for $R^{t} R$ to be invertible by a differential operator.

It is surprising to realize that in most cases-when $1 / \sigma\left(R^{t} R\right)$ is not a polynomial-one needs to know integrals of $f \in C_{0}^{\infty}(X)$ over $H_{y}$ that are far from $x \in X$ to recover $f(x)$ from $R f$.

3. The Radon transform on $\mathbf{R}^{n}$ with arbitrary measures. We now apply the results of the last section to the double fibration of Example 1. First we show that this fibration satisfies the Bolker assumption and so $R^{t} R$ is an elliptic pseudodifferential operator. Then we calculate the symbol of $R^{t} R$ in terms of the defining measures (Theorem 3.1).

Recall the notation of Example 1. Let $X=\mathbf{R}^{n}$ and let $Y$ be the set of hyperplanes in $\mathbf{R}^{n}$. If $\omega \in \mathbf{S}^{n-1}$ and $p \in \mathbf{R}$, then the hyperplane $H(\omega, p)=\left\{x \in \mathbf{R}^{n} \mid x \cdot \omega\right.$ $=p$ \} has normal vector $\omega$ and directed distance $p$ from the origin. Because 
$H(\omega, p)=H(-\omega,-p), Y$ can be identified with $\left(\mathbf{S}^{n-1} \times \mathbf{R}\right) / \mathbf{Z}_{2}$. Then

$$
Z=\left\{(x,[\omega, x \cdot \omega]) \mid x \in \mathbf{R}^{n}, \omega \in \mathbf{S}^{n-1}\right\}
$$

is diffeomorphic to $\mathbf{R}^{n} \times \mathbf{R P}^{n-1}$.

Let $d \omega d p$ and $d x d \omega$ be the standard measures of Example 1 on $Y$ and $Z$, respectively and let $d x$ be Lebesgue measure on $X$. Choose positive smooth measures

$$
\begin{array}{ll}
\mu=\mu(x,[\omega, x \cdot \omega]) d x d \omega & \text { on } Z \\
m=m(x) d x & \text { on } X \\
n=n([\omega, p]) d \omega d p & \text { on } Y .
\end{array}
$$

These measures define a Radon transform and its dual.

Let $(x, \xi) \in T^{*} X-0$ where $\xi=|\xi| \omega \cdot \mathbf{d x}(|\xi|$ is the norm of $\xi$ in the standard metric on $T^{*} X, \omega=\left(\omega_{1}, \ldots, \omega_{n}\right) \in S^{n-1}$ and $\omega \cdot d x$ is defined to be $\sum_{j-1}^{n} \omega_{j} d x_{j} \in$ $\left.T_{x}^{*} \mathbf{R}^{n}\right)$. The following theorem is a special case of Theorem 2.1.

THEOREM 3.1. With measures given in (16) for the Radon transform on $\mathbf{R}^{n}, R^{t} R$ is an elliptic pseudodifferential operator of order $1-n$ and, for $(x, \xi) \in T^{*} \mathbf{R}^{n}-0$, $\xi=|\xi| \omega \cdot \mathbf{d x}$,

$$
\sigma\left(R^{t} R\right)(x, \xi)=\frac{(2 \pi)^{n-1} \mu^{2}(x,[\omega, x \cdot \omega])}{m(x) n([\omega, x \cdot \omega])|\xi|^{n-1}} .
$$

As an illustration we calculate the symbol of $R^{t} R$ when the classical measures of Example 1 are used. In that example, $\mu=d x d \omega, m=d x$ and $n=d \omega d p$ so $\sigma\left(R^{t} R\right)(x, \xi)=(2 \pi)^{n-1} /|\xi|^{n-1}$.

In the general case, with measures given by (16), Theorems 2.2 and 3.1 conclude that $R^{t} R$ is not invertible by a differential operator if $m(x) n([\omega, x$. $\omega])|\xi|^{n-1} / \mu^{2}(x,[\omega, x \cdot \omega])$ is not a polynomial in $\xi(\xi=|\xi| \omega \cdot \mathbf{d x})$.

Proof of Theorem 3.1. Let $\Gamma=N^{*} Z-0$. The proof consists of showing that this fibration satisfies the Bolker assumption then choosing local coordinates and using (15) to calculate $\sigma\left(R^{t} R\right)$.

It will be convenient to identify $T_{[\omega, p]}^{*} Y$ with $T_{(\omega, p)}^{*}\left(\mathbf{S}^{n-1} \times \mathbf{R}\right)$ and $N_{(x,[\omega, x \cdot \omega]}^{*} Z$ with the appropriate subspace of $T_{(x, \omega, x \cdot \omega)}^{*}\left(\mathbf{R}^{n} \times \mathbf{S}^{n-1} \times \mathbf{R}\right)$. If $x \in \mathbf{R}^{n}$ we define $J x=x-(x \cdot \omega) \omega$. This is the projection of $x$ to the hyperplane $H(\omega, 0)$ perpendicular to $\omega$. The hyperplane will be identified with $T_{\omega}^{*} S^{n-1}$ and the element corresponding to $J x$ will be denoted $J x \cdot \mathrm{d} \omega$. With these identifications one can calculate that

$$
\begin{aligned}
& \Gamma=\left\{(x,[\omega, p] ; a(J x \cdot \mathrm{d} \omega+\omega \cdot \mathrm{dx}-\mathrm{dp})) \mid(x, \omega, p) \in \mathbf{R}^{n} \times \mathbf{S}^{n-1}\right. \times \mathbf{R}, \\
&x \cdot \omega=p, a \in \mathbf{R}-0\} .
\end{aligned}
$$

Using this expression for $\Gamma$, it is straightforward to show that $\rho: \Gamma \rightarrow T^{*} Y$ is a 1-to-1 immersion. This is left as an exercise. Therefore the Bolker assumption holds and we now apply Theorem 2.1 to calculate $\sigma\left(R^{t} R\right)$. 
Let $\xi \in T_{x}^{*} \mathbf{R}^{n}$; by a rotation we can assume $\xi=a e_{n} \cdot \mathbf{d x}=a d x_{n}(a=|\xi|)$ in which case the expression for $\Gamma$ verifies that $\pi^{-1}(x, \xi)=\left(x,\left[e_{n}, x_{n}\right] ; a(J x \cdot d \omega+\right.$ $\left.\left.\mathbf{d x}_{n}-\mathbf{d p}\right)\right)$.

Let $\tilde{S}=\left\{\omega \in \mathbf{S}^{n-1} \mid \omega_{n}>0\right\}$ be an open hemisphere of $\mathbf{S}^{n-1}$. Let $\tilde{Y}=\tilde{S} \times \mathbf{R}$ and $\tilde{Z}=\left\{(x, \omega, x \cdot \omega) \mid x \in \mathbf{R}^{n}, \omega \in \tilde{S}\right\}$; then $\tilde{Z}$ is diffeomorphic to $\mathbf{R}^{n} \times \tilde{S}$ and $\tilde{Z}$ inherits its "standard measure" from the standard measure on $\mathbf{R}^{n} \times \tilde{S}$ (i.e., the one coming from the Riemannian structure). The maps $\tilde{Y} \rightarrow Y,(\omega, p) \rightarrow[\omega, p]$ and $\tilde{Z} \rightarrow Z,(x, \omega, x \cdot \omega) \rightarrow(x,[\omega, x \cdot \omega])$, give coordinates on $Y$ and $Z$ such that the measures $d \omega d p$ and $d x d \omega$ of (16) agree with the standard measures on the coordinate neighborhoods. Define $w(x, \omega, p)=x \cdot \omega-p$; then $\tilde{Z}$ is defined in $X \times \tilde{Y}$ by the equation $w=0$. Therefore, by (15),

$$
\sigma\left(R^{t} R\right)(x, \xi)=\frac{(2 \pi)^{n-1} \mu^{2}(x,[\omega, x \cdot \omega])}{m(x) n([\omega, x \cdot \omega])} \frac{d x^{2} d \omega^{2} d w d \eta}{d x d \omega d p \pi^{*}\left|\sigma_{X}\right|^{n / 2} \rho^{*}\left|\sigma_{Y}\right|^{n / 2}}
$$

where $d \eta$ is the density on the fiber of $\tilde{\Gamma}=N^{*} \tilde{Z}-0$ dual to $d w$ (see below (11)).

To finish the proof of the theorem we only need to apply the following lemma to (17).

LeMMA 3.2. At $\lambda=\pi^{-1}(x, \xi)$,

$$
\frac{d x^{2} d \omega^{2} d w d \eta}{d x d \omega d p \pi^{*}\left|\sigma_{X}\right|^{n / 2} \rho^{*}\left|\sigma_{Y}\right|^{n / 2}}=\frac{1}{|\xi|^{n-1}} .
$$

Proof. It is straightforward to show that the measures $d x d \omega d p$ and $d x d \omega d w$ agree on $X \times \tilde{Y}$ and so the left-hand side of (18) becomes $d x d \omega d \eta / \pi^{*}\left|\sigma_{X}\right|^{n / 2} \rho^{*}\left|\sigma_{Y}\right|^{n / 2}$. Because $\xi=|\xi| e_{n} \cdot \mathbf{d x}$, a basis $B$ of $T_{\lambda} \Gamma$ is easy to calculate. By evaluating the densities $d x d \omega d \eta$ and $\pi^{*}\left|\sigma_{X}\right|^{n / 2} \rho^{*}\left|\sigma_{Y}\right|^{n / 2}$ on $B$ one shows

$$
|\xi|^{n-1} d x d \omega d \eta=\pi^{*}\left|\sigma_{X}\right|^{n / 2} \rho^{*}\left|\sigma_{Y}\right|^{n / 2},
$$

which proves the lemma and the theorem.

Similar calculations have been performed for the Radon transforms with arbitrary measures on points and complex hyperplanes on $\mathbf{C}^{n}$ and points and projective hyperplanes on $\mathbf{R P}^{n}$ and $\mathbf{C P}^{n}$ [17]. Their symbols are quite similar to the result of Theorem 3.1.

4. Translation invariant Radon transforms on $\mathbf{R}^{n}$. In this section we investigate the Radon transform on points and hyperplanes in $R^{n}$. We assume that $R^{t} R$ is a translation invariant operator (i.e., a convolution operator). There are several reasons to study these transforms; they are natural generalizations of the classical Radon transform on $\mathbf{R}^{n}$ which is both translation and rotation invariant. As we shall see, these operators are invertible and have other nice properties.

We first give the restrictions that translation invariance puts on the defining measures (Proposition 4.1). Then we quote a result about Fourier transforms and use this to invert $R^{t} R$ and to prove the converse of Theorem 2.2; for these transforms the inverse to $R^{t} R$ is a differential operator if and only if $1 / \sigma\left(R^{t} R\right)$ is a 
polynomial (Theorem 4.2). Because we know the symbol of $R^{t} R$ in terms of the measures (Theorem 3.1), this is a necessary and sufficient condition on the defining measures for $R^{t} R$ to be invertible by a differential operator.

Let $X, Y$, and $Z$ be defined as in Example 1 and let

$$
\begin{aligned}
\mu & =2 \mu(x,[\omega, x \cdot \omega]) d x d \omega, \\
m & =m(x) d x . \\
n & =2 n([\omega, p]) d \omega d p,
\end{aligned}
$$

be positive $C^{\infty}$ measures on $Z, X$ and $Y$, respectively. The measures $d x d \omega$ and $d \omega d p$ are the canonical measures on $Z$ and $Y$ from Example 1.

We now calculate the Radon transform $R$ and its dual $R^{t}$ induced from the measures (19). Let $\mu(x, \omega, x \cdot \omega)$ be the function $\mu$ in (19) considered as a function on $\mathbf{R}^{n} \times \mathbf{S}^{n-1}$ that is even in $\omega$. Let $n(\omega, p)$ be the function $n([\omega, p])$ considered as an even function on $\mathbf{S}^{n-1} \times \mathbf{R}$ and let $d x_{H}$ be Lebesgue measure on the hyperplane $H(\omega, p)$. Because $d x_{H} d \omega d p$ and $d x d \omega$ define the same measure on $Z$, the measure on $H(\omega, p)$ coming from (19) is

$$
d \mu_{[\omega, p]}=\frac{\mu(x, \omega, p)}{n(\omega, p)} d x_{H} .
$$

Therefore for $f \in C_{0}^{\infty}\left(\mathbf{R}^{n}\right)$ we see from (5) that

$$
R f(\omega, p)=\int_{H(\omega, p)} \frac{f(x) \mu(x, \omega, p)}{n(\omega, p)} d x_{H} .
$$

One can calculate the measure $\mu_{x}$ on $G_{x}$ in a similar manner. Let $d \omega_{S}$ be the standard measure on $\mathbf{S}^{n-1}$; then it is straightforward to calculate the pullback of $\mu_{x}$ under the 2-to-1 cover $\mathbf{S}^{n-1} \rightarrow G_{x}, \omega \rightarrow[\omega, x \cdot \omega]$. One concludes that, for $g \in$ $C^{\infty}(Y)$,

$$
R^{t} g\left(x_{0}\right)=\int_{S^{n-1}} g\left(\left[\omega, x_{0} \cdot \omega\right]\right) \frac{\mu\left(x_{0}, \omega, x_{0} \cdot \omega\right)}{m\left(x_{0}\right)} d \omega_{S}
$$

The proof of our next proposition will help to invert $R^{t} R$.

Proposition 4.1. With measures given in (19), $R^{t} R$ is translation invariant if and only if

$$
\frac{\mu^{2}(x, \omega, x \cdot \omega)}{m(x) n(\omega, x \cdot \omega)}=a(\omega)
$$

is a function of $\omega$ only, and

$$
m(x)=m(0) e^{2 z \cdot x} \quad \text { for some } z \in \mathbf{R}^{n} .
$$

Proof. $R^{t} R$ is calculated for general measures and evaluated on specific distributions from which the proposition is deduced. We first prove the necessity of (22) and (23). Let $K$ be $S O(n)$ and let $L$ be the isotropy subgroup of $e_{n}$. Let $M$ be the isotropy subgroup of $e_{1}$ and let $d k, d l$, and $d m$ be the invariant measures on these groups that give total measure one. Define $\Omega_{q}$ to be the volume of $\mathbf{S}^{q}$ under its standard metric. 
Let $S^{n-2}=\left\{\omega \in \mathbf{S}^{n-1} \mid \omega_{1}=0\right\}$ be a great sphere of $\mathbf{S}^{n-1}$. Because $M$ fixes $e_{1}$, $M S^{n-2}=S^{n-2}$ and if $k \in K$ and $h \in C^{\infty}\left(\mathbf{S}^{n-1}\right)$,

$$
\hat{h}\left(k e_{1}\right)=\Omega_{n-2} \int_{M} h\left(k m e_{n}\right) d m
$$

is the integral of $h$ over the great sphere $\mathbf{S}^{n-1} \cap H\left(k e_{1}, 0\right)$ in its standard measure. Because $h \rightarrow \hat{h}$ is the pullback to $\mathbf{S}^{n-1}$ of a classical Radon transform on $\mathbf{R P}^{n-1}$, this map is invertible for even functions $h$ ([1], [9], [21]). For similar reasons if $q \in C_{0}^{\infty}\left(\mathbf{R}^{n}\right)$ and $x_{0} \in H\left(k e_{n}, p\right)$,

$$
\int_{H\left(k e_{n}, p\right)} q(x) d x_{H}=\Omega_{n-2} \int_{0}^{\infty} r^{n-2} \int_{L} q\left(x_{0}+r k l e_{1}\right) d l d r
$$

is the integral of $q$ over $H\left(k e_{n}, p\right)$ expressed in spherical coordinates.

Let $f \in C_{0}^{\infty}\left(\mathbf{R}^{n}\right)$ and let $x_{0} \in H\left(k e_{n}, p\right)$, then using (25) in (20) we can rewrite $R f\left(k e_{n}, p\right)$. Substituting $k e_{n}$ for $\omega$ and $\Omega_{n-1} d k$ for $d \omega_{s}$ in (21) and composing the result with the new expression for $R f\left(k e_{n}, p\right)$ we find that

$$
\begin{aligned}
& R^{t} R f\left(x_{0}\right)= \Omega_{n-1} \Omega_{n-2} \\
& \cdot \int_{K} \int_{0}^{\infty} \int_{L}\left\{r^{n-2} f\left(x_{0}+r k l e_{1}\right) \frac{\mu\left(x_{0}+r k l e_{1}, \omega, x_{0} \cdot \omega\right)}{n\left(\omega, x_{0} \cdot \omega\right)}\right. \\
&\left.\cdot \frac{\mu\left(x_{0}, \omega, x_{0} \cdot \omega\right)}{m\left(x_{0}\right)}\right\}\left.\right|_{\omega=k e_{n}} d l d r d k .
\end{aligned}
$$

Let

$$
a^{\prime}(x, y, \omega)=\frac{\mu(x+y, \omega, x \cdot \omega) \mu(x, \omega, x \cdot \omega)}{m(x) n(\omega, x \cdot \omega)}
$$

for $y \cdot \omega=0$; we write the integral above using $a^{\prime}$ and then use Fubini's theorem [19] to reverse integrations with respect to $d l$ and $d k$. Finally using the right invariance of $d k$, we substitute $k l^{-1}$ for $k$ and discover that

$$
R^{t} R f\left(x_{0}\right)=\Omega_{n-1} \Omega_{n-2} \int_{0}^{\infty} \int_{K} r^{n-2} f\left(x_{0}+r k e_{1}\right) a^{\prime}\left(x_{0}, r k e_{1}, k e_{n}\right) d k d r .
$$

Let $d k_{M}$ be the $K$ invariant measure on $K / M$ satisfying

$$
\int_{K} g(k) d k=\int_{K / M} \int_{M} g(k m) d m d k_{M} \text { for } g \in C^{\infty}(K) .
$$

Using this identity, we change the last expression. Recall that $M$ is the isotropy group of $e_{1}$. Then

$$
\begin{aligned}
R^{t} R f\left(x_{0}\right)=\Omega_{n-1} \int_{0}^{\infty} \int_{K / M} r^{n-2} f\left(x_{0}+\right. & \left.r k e_{1}\right) \Omega_{n-2} \\
& \times \int_{M} a^{\prime}\left(x_{0}, r k e_{1}, k m e_{n}\right) d m d k_{M} d r
\end{aligned}
$$


The inner integral over $M$ is just $\hat{a}^{\prime}\left(x_{0}, r k e_{1}, k e_{1}\right)$ (see (24)). By changing back to rectangular coordinates $\left(y=r k e_{1}\right)$ we see that

$$
R^{t} R f\left(x_{0}\right)=\int_{\mathbf{R}^{n}} f\left(x_{0}+y\right) \frac{\hat{a}^{\prime}\left(x_{0}, y, y /|y|\right)}{|y|} d y .
$$

Assume $R^{t} R$ is translation invariant; we first prove (22). Let $\chi_{\omega, e}$ be an approximate identity about $\omega \in \mathbf{S}^{n-1}$ (that is $\chi_{\omega, e} \in C^{\infty}\left(\mathbf{S}^{n-1}\right.$ ) and $\lim _{\varepsilon \rightarrow 0} \chi_{\omega, e}=\delta_{\omega}$, the Dirac delta function on $S^{n-1}$ at $\omega$ ). Let $\varphi_{e}$ be an approximate identity at $0 \in R$ such that $\varphi_{e}(r)$ is 0 in a neighborhood of $(-\infty, 0]$. Finally let $\omega_{0} \in \mathbf{S}^{n-1}, x_{0} \in \mathbf{R}^{n}$ and define

$$
\psi_{\omega_{0, e, x_{0}}}\left(x_{0}+y\right)=\frac{\chi_{\omega_{0, e}}(y /|y|) \varphi_{e}(|y|)}{|y|^{n-2}} .
$$

Using equation (27) we see that $\lim _{e \rightarrow 0} R^{t} R \psi_{\omega_{0, e}, x_{0}}\left(x_{0}\right)=\hat{a}^{\prime}\left(x_{0}, 0, \omega_{0}\right)$. By the assumption that $R^{t} R$ is translation invariant, this limit is equal to

$$
\lim _{\varepsilon \rightarrow 0} R^{t} R \psi_{\omega_{0, e}, 0}(0)=\hat{a}^{\prime}\left(0,0, \omega_{0}\right) .
$$

For a fixed $x$ the function $a^{\prime}(x, 0, \omega)$ is an even function of $\omega$. Because the transform (24) is invertible on even functions of $\omega$ ([9], [21]) and $\hat{a}^{\prime}(x, 0, \omega)=$ $\hat{a}^{\prime}(0,0, \omega)$ we see that $a^{\prime}(x, 0, \omega)=a^{\prime}(0,0, \omega)$ is a function not dependent on $x$. Let $a(\omega)=a^{\prime}(x, 0, \omega)$; then the expression (26) for $a^{\prime}$ proves (22).

Let $\delta_{x_{0}+r \omega}$ be the Dirac delta function at $x_{0}+r \omega \in \mathbf{R}^{n}, r \neq 0, \omega \in \mathbf{S}^{n-1}$, and let

$$
\varphi_{x_{0}+r \omega}\left(x_{0}+y\right)=|y| \delta_{x_{0}+r \omega}\left(x_{0}+y\right) \text {. }
$$

By (28) and the translation invariance of $R^{t} R$ :

$$
\begin{aligned}
R^{t} R \varphi_{x_{0}+r \omega}\left(x_{0}\right) & =\hat{a}^{\prime}\left(x_{0}, r \omega, \omega\right)=R^{t} R \varphi_{r \omega}(0) \\
& =\hat{a}^{\prime}(0, r \omega, \omega) .
\end{aligned}
$$

Solving for $\mu$ in (22) shows that

$$
\mu(x, \omega, x \cdot \omega)=\sqrt{a(\omega) m(x) n(\omega, x \cdot \omega)} .
$$

If we use (29) in (26) and take the transform (24), we find

$$
\begin{aligned}
\hat{a}^{\prime}\left(x_{0}, r \omega, \omega\right) & =\hat{a}(\omega)\left(\frac{m\left(x_{0}+r \omega\right)}{m\left(x_{0}\right)}\right)^{1 / 2}, \\
\hat{a}^{\prime}(0, r \omega, \omega) & =\hat{a}(\omega)\left(\frac{m(r \omega)}{m(0)}\right)^{1 / 2}
\end{aligned}
$$

Because $\hat{a}^{\prime}\left(x_{0}, r \omega, \omega\right)=\hat{a}^{\prime}(0, r \omega, \omega)$ is never zero, the right-hand sides of (30) can be manipulated to show that the map $x \rightarrow m(x) / m(0)$ is a continuous homomorphism from $\left(\mathbf{R}^{n},+\right)$ to $\left(\mathbf{R}^{+}, \cdot\right)$ and so is of the form $m(x) / m(0)=e^{2 z \cdot x}$ for some $z \in \mathbf{R}^{n}$. This proves (23). 
Assume (22) and (23) hold. Inserting the expression (29) into the definition (26) of $a^{\prime}$, and using this in (28) shows that

$$
\begin{aligned}
R^{t} R f(x) & =\int_{\mathbf{R}^{n}} f(x+y) \frac{\hat{a}(y /|y|) e^{z \cdot y}}{|y|} d y \\
& =e^{-x \cdot z} \int_{\mathbf{R}^{n}} f(x+y) e^{z \cdot(x+y)} \frac{\hat{a}(y /|y|)}{|y|} d y
\end{aligned}
$$

for $f \in C_{0}^{\infty}\left(\mathbf{R}^{n}\right)$. This is a convolution operator and therefore is translation invariant. This finishes the proof of the proposition.

One fact about Fourier transforms is needed before $R^{t} R$ can be inverted. First we define the Fourier transform $F$ and its dual $F^{t}$. If $f \in C_{0}^{\infty}\left(R^{n}\right)$ we let

$$
\begin{aligned}
F f(\xi) & =\int_{\mathbf{R}^{n}} e^{-i \xi \cdot x} f(x) d x, \\
F^{t} f(x) & =\int_{\mathbf{R}^{n}} e^{i x \cdot \xi} f(\xi) d \xi
\end{aligned}
$$

It is shown in [21] that

$$
F^{t}\left(\frac{a(\xi /|\xi|)}{|\xi|^{n-1}}\right)(x)=(\pi /|x|) \hat{a}(x /|x|)
$$

The proof uses techniques in [5] and [6] to evaluate the Fourier transform of an even homogeneous function on $\mathbf{R}^{n}$. This implies that

$$
F\left(\frac{\hat{a}(x /|x|)}{|x|}\right)(\xi)=2^{n} \pi^{n-1} \frac{a(\xi /|\xi|)}{|\xi|^{n-1}}
$$

Using (32) we can write $R^{t} R$ as a pseudodifferential operator. Let $f \in C_{0}^{\infty}\left(\mathbf{R}^{n}\right)$. The second integral in (31) is a convolution, so taking Fourier transforms and then inverse transforms of that integral proves

$$
R^{t} R f(x)=\frac{e^{-x \cdot z}}{(2 \pi)^{n}} \int_{R^{n}} e^{i x \cdot \xi} \frac{2 a(\xi /|\xi|)(2 \pi)^{n-1}}{|\xi|^{n-1}} F\left(e^{z} \cdot f\right)(\xi) d \xi
$$

where $e^{z \cdot}(x)=e^{z \cdot x}$.

By the definition (22) of $a$, the symbol of $R^{t} R$ given in Theorem 3.1 for the measures in (19) is

$$
\sigma\left(R^{t} R\right)(x, \xi)=\frac{2 a(\xi /|\xi|)(2 \pi)^{n-1}}{|\xi|^{n-1}}=\left.\frac{2 \mu^{2}(x, \omega, x \cdot \omega)(2 \pi)^{n-1}}{m(x) n(\omega, x \cdot \omega)|\xi|^{n-1}}\right|_{\omega=\xi / \xi \mid} .
$$

Define the pseudodifferential operator $\boldsymbol{A}$ as follows:

$$
A f(x)=\frac{e^{-z \cdot x}}{(2 \pi)^{n}} \int_{\mathbf{R}^{n}} e^{i x \cdot \xi} \frac{|\xi|^{n-1}}{2 a(\xi /|\xi|)} \frac{F\left(e^{z \cdot} \cdot f\right)(\xi)}{(2 \pi)^{n-1}} d \xi .
$$

It is straightforward to check that $A$ inverts $R^{t} R$. The definition of $A$ shows that $A$ is a differential operator if and only if $1 / \sigma\left(R^{t} R\right)$ is a polynomial in $\xi$. This proves our final theorem. 
THEOREM 4.2. With measures given by (19) assume that $R^{t} R$ is a translation invariant operator. Then for $f \in C_{0}^{\infty}\left(\mathbf{R}^{n}\right)$,

(i)

$$
R^{t} R f(x)=\left.\frac{e^{-x \cdot z}}{(2 \pi)^{n}} \int_{\mathbf{R}^{n}} e^{i x \cdot \xi} \frac{2 \mu^{2}(x, \omega, x \cdot \omega)(2 \pi)^{n-1}}{m(x) n(\omega, x \cdot \omega)|\xi|^{n-1}}\right|_{\omega=\xi /|\xi|} F\left(e^{z \cdot f} f\right)(\xi) d \xi,
$$

(ii) $R^{t} R$ is invertible by $A$ given in (33) and $A$ is a differential operator if and only if

$$
\frac{1}{\sigma\left(R^{t} R\right)(x, \xi)}=\left.\frac{m(x) n(\omega, x \cdot \omega)|\xi|^{n-1}}{2 \mu^{2}(x, \omega, x \cdot \omega)(2 \pi)^{n-1}}\right|_{\omega=\xi / \xi \mid}
$$

is a polynomial in $\xi$.

For translation invariant transforms, this theorem shows explicitly how $R^{t} R$ depends on the defining measures and how invertibility by a differential operator is affected by the defining measures. These results are apparent benefits of expressing $R^{\prime} R$ as a pseudodifferential operator. Because the classical Radon transform on $\mathbf{R}^{n}$ (Example 1) is translation invariant, Theorem 4.2 also shows that it is invertible.

\section{REFERENCES}

1. P. Funk, Über eine geometrische Anwendung der Abelschen Integralgleichung, Math. Ann. 77 (1916), 129-135.

2. I. M. Gelfand and M. I. Graev, The geometry of homogeneous spaces, group representations in homogeneous spaces and questions in integral geometry related to them, Amer. Math. Soc. Transl. 37 (1964), 351-429.

3. I. M. Gelfand, M. I. Graev and Z. Ya. Shapiro, Differential forms and integral geometry, Functional Anal. Appl. 3 (1969), 24-40.

4. I. M. Gelfand, M. I. Graev and N. Ya. Vilenkin, Generalized functions, Vol. 5, Academic Press, New York, 1966.

5. I. M. Gelfand and Z. Ya. Shapiro, Homogeneous functions and their extensions, Amer. Math. Soc. Transl. (2) 8 (1958), 21-86.

6. I. M. Gelfand and G. E. Shilov, Generalized functions, Vol. 1, Academic Press, New York, 1964.

7. V. Guillemin, On some results of Gelfand in integral geometry, Lecture notes, Symposium on Global Analysis, Durham, N. C., July 1976.

8. V. Guillemin and S. Sternberg, Geometric asymptotics, Math. Surveys 14, Amer. Math. Soc., Providence, R. I., 1977.

9. S. Helgason, Differential operators on homogeneous spaces, Acta Math. 102 (1959), 239-299.

10. $\quad$ A duality in integral geometry; some generalizations of the Radon transform, Bull. Amer. Math. Soc. 70 (1964), 435-446.

11. The Radon transform on Euclidean spaces, compact two-point homogeneous spaces and Grassmann manifolds, Acta Math. 113 (1965), 153-180.

12. , A duality for symmetric spaces, with applications to group representations, Advances in Math. 5 (1970), 1-154.

13. _ The surjectivity of invariant differential operators on symmetric spaces. I, Ann. of Math. 98 (1973), 451-479.

14. L. Hormander, Fourier integral operators. I, Acta Math. 127 (1971), 79-183.

15. F. John, Bestimmung einer Funktion aus ihren Integralen über gewisse Mannigfaltigkeiten, Math. Ann. 100 (1934), 488-520.

16. D. Ludwig, The Radon transform on Euclidean space, Comm. Pure Appl. Math. 69 (1966), 49-81. 
17. E. Quinto, On the locality and invertibility of Radon transforms, $\mathrm{Ph}$. D. Thesis, M.I.T., Cambridge, Mass., 1978.

18. J. Radon, Über die Bestimmung von Funktionen durch ihre Integralwerte längs gewisser Mannigfalligkeiten, Ber. Verh. Sächs. Akad. 69 (1917), 262-277.

19. W. Rudin, Real and complex analysis, McGraw-Hill, New York, 1966.

20. V. I. Semyanistyi, On some integral transforms in Euclidean space, Soviet Math. Dokl. 1 (1960), 1114-1117.

21.

, Homogeneous functions and some problems of integral geometry in spaces of constant curvature, Soviet Math. Dokl. 2 (1961), 59-62.

Department of Mathematics, Tufts University, Medford, Massachusetts 02155 